\title{
Simulation of AlGaN/GaN HEMTs' Breakdown Voltage Enhancement Using Grating Field Plates
}

\author{
E. Bahat-Treidel, V. Sidorov, J. Würfl, and G. Tränkle \\ Ferdinand-Braun-Institut für Hoechstfrequenztechnik, Gustav-Kirchhoff-Strasse 4, \\ 12489 Berlin - Germany \\ eldad.bahat-treidel@fbh-berlin.de
}

\begin{abstract}
Two dimensional physical-based device simulations (Silvaco - "Atlas") of breakdown voltage $\left(V_{b r}\right)$ effect in AlGaN/GaN HEMTs (high electron mobility transistors) on silicon carbide $(4 \mathrm{H}-\mathrm{SiC})$ device are preformed. The influence of novel single layer grating field plates (FPs), consisting of gate connected fingers and floating fingers, on $V_{b r}$, obtained via the electric field distribution in the AlGaN layer is studied. We have found that the grating FPs reduce the electric field peaks and efficiently distribute the electrical filed along the AlGaN-layer and thus enhance device $V_{b r}$ characteristics.
\end{abstract}

\section{Introduction}

Breakdown voltage enhancement in HEMTs and other field effect devices, using field plates (FPs) is a well known method [1-3]. In HEMTs the FPs distribute the electric field in the channel more equally and by that improve the device performance. In this case the electric field is reduced in critical volumes such as under the gate's drain side and under the drain's gate side. Continuous FPs generate a single electric field peak under the edges of the FPs [4;5] and limit their efficiency to "flatten" the field in critical volumes of the device. In order to overcome this problem the idea of multiple stacked FPs [6] has been developed. They improve the device breakdown $V_{b r}$ performance but have major drawbacks such as multi-layer processing thus requiring more photolithographic cycles. This is associated with a dramatic increase of the device capacitance limiting its high frequency performance. Therefore we are suggesting single layer grating FPs (Fig. 1.). They could utilize the same mask as the gate, so no additional process cycles are required. Prior to device processing we have demonstrated the advantages of the grating FPs on AlGaN/GaN HEMTs devices using Silvaco ATLAS [7] simulation. Additional advantages of the grating FP are associated with the flexibility of connecting the grating "fingers" to different potentials sources such as the gate, the drain, external potential, and floating fingers to reduce the device capacitance. 


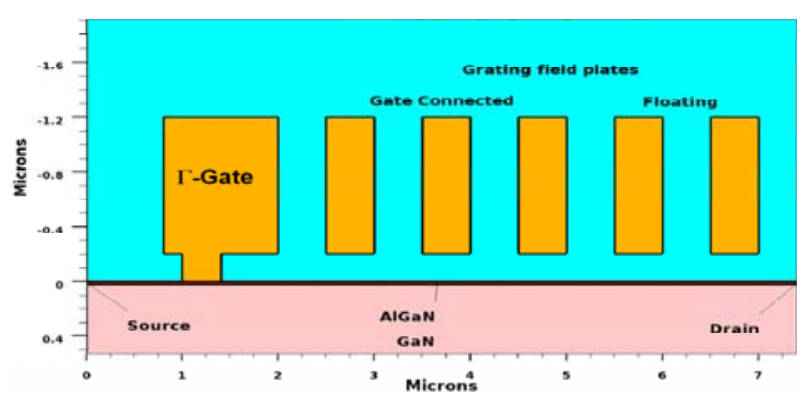

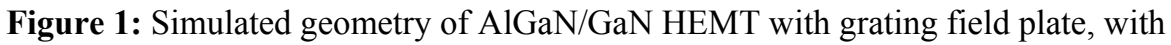
three gate connected fingers and two floating fingers.

\section{Device model, Simulation and Discussion}

Example configurations of AlGaN/GaN HEMTs with grating FPs were chosen to emphasize enhanced $V_{b r}$ capabilities. The Chuang's three band model in wurtzite materials and band parameter models in drift diffusion was chosen for the electronic band-structure, Albrecht low field mobility model was chosen for modelling of carrier mobility [7]. By implementing spontaneous polarization charges, piezoelectric strains, interface charges the two dimension electron gas (2DEG) high mobility layer was modelled. Bulk traps at discrete energy levels within the band-gap of the semiconductor and a fixed oxide charge density are additionally applied at a semiconductor / insulator interface to keep the system electrically neutral. The breakdown voltage simulation was based on the self-heating simulator. In order to reduce computation resources only dominating carriers i.e. electrons were considered (no impact ionization was calculated). The basic device simulated consists of an embedded gate architecture with a $0.6 \mu \mathrm{m}(\mathrm{Gamma}) \Gamma$-gate FP configuration (a, see also Fig. 1.). To this device we added grating FPs with three gate connected fingers (b) in same processing layer, or with two gate connected fingers and two floating fingers (c), or with three gate connected fingers and two floating fingers (d). The devices $V_{b r}$ were obtained by simulation. FP arrangement (d) resulted in a $35 \%$ increase of $V_{b r}$ and therefore confirmed our basic assumptions (Fig. 2.). The distribution of the electric field at $V_{d s}=V_{b r}$ in the active layer reveals it's "flattened" characteristic relative to the basic device's field (Fig. 3.). At a high drain voltage close to the $V_{b r}$ the intensity of the field under the drain's gate side is very high. Grated FP architectures have the potential to shift this field maximum away from the drain contact into the bulk semiconductor region and therefore influence breakdown voltage (Fig. 4.). 


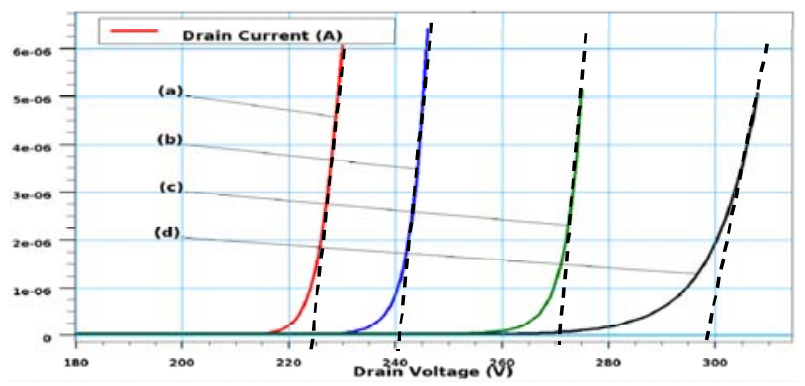

Figure 2: Breakdown voltage characteristics of Simulated AlGaN/GaN HEMTs: (a) no grating FPs, (b) with grating FP consists of three gate connected fingers, (c) with grating FP consists of two gate connected fingers and two floating fingers, (d) with grating FP consists of three gate connected fingers and two floating fingers.

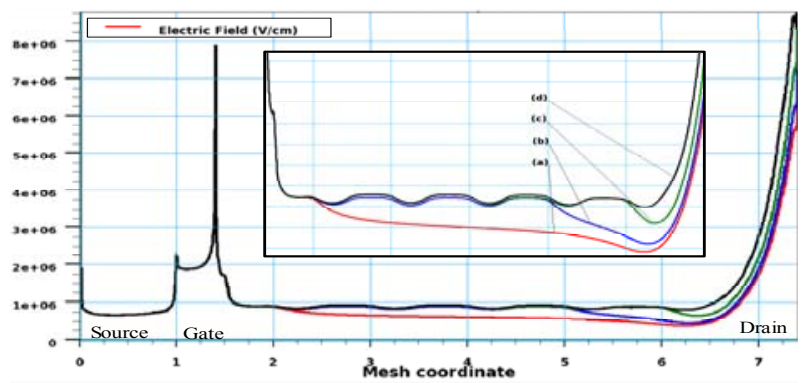

Figure 3: Electric field characteristics of Simulated AlGaN/GaN HEMTs at the active layer at $V_{d s}=V_{b r}$ : (a) no grating FPs, (b) with grating FP consists of three gate connected fingers, (c) with grating FP consists of two gate connected fingers and two floating fingers, (d) with grating FP consists of three gate connected fingers and two floating fingers.

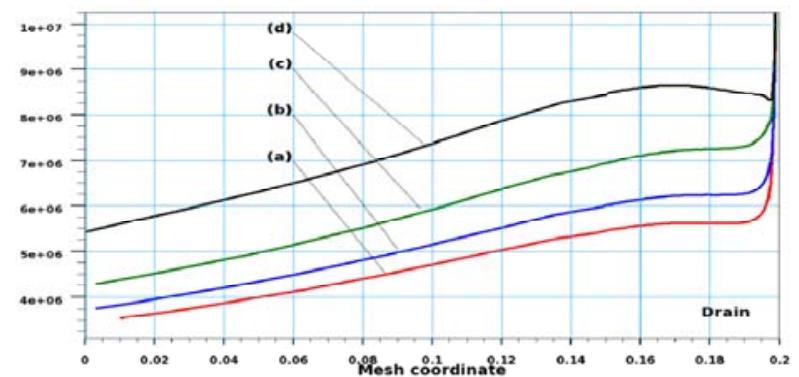

Figure 4: Insight of the drain electric field characteristics of Simulated AlGaN/GaN HEMTs at the active layer at $V_{d s}=V_{b r}$ : (a) no grating FPs, (b) with grating FP consists of three gate connected fingers, (c) with grating FP consists of two gate connected fingers and two floating fingers, (d) with grating FP consists of three gate connected fingers and two floating fingers. 


\section{Conclusion}

Grating FPs enable control and shaping of the electric field profile in field effect devices. In $\mathrm{Al}-\mathrm{GaN} / \mathrm{GaN}$ HEMTs they increase the field magnitudes between the gate and the drain on the expense of the gate and drain electric field peaks and thus reduce field gradients. This reduction leads to an improvement of $V_{b r}$ which has been demonstrated by physical simulation. Grating FPs are easy to manufacture with the current $\mathrm{AlGaN} / \mathrm{GaN}$ HEMT process technologies with no additional coasts. Devices processed currently will put more light on the capabilities of this approach. Further investigation is done on the device geometrical optimization such as different biasing sources and on the device frequency characteristics.

\section{References}

[1] S. Karmalkar, D. Jianyu, and M. S. Shur, "RESURF Al-GaN/GaN HEMT for high voltage power switching", IEEE Electron Device Lett., vol. 22, no. 8, pp. 373-375, 2001.

[2] A. Chini, D. Buttari, R. Coffie, S. Heikman, S. Keller, and U. K. Mishra, "12 W/mm power density AlGaN/GaN HEMTs on sapphire substrate", Electronics Letters, vol. 40, no. 1, pp. 73-74, 2004.

[3] Y. Okamoto, Y. Ando, T. Nakayama, K. Hataya, H. Miya-moto, T. Inoue, M. Senda, K. Hirata, M. Kosaki, N. Shi-bata, and M. Kuzuhara, "High-power recessed-gate AlGaNGaN HFET with a field-modulating plate", Electron Devices, IEEE Transactions on, vol. 51, no. 12, pp. 2217-2222, 2004.

[4] S. Karmalkar and U. K. Mishra, "Enhancement of breakdown voltage in AlGaN/GaN high electron mobility transistors using a field plate", Electron Devices, IEEE Transactions on, vol. 48, no. 8, pp. 1515-1521, 2001.

[5] W. Saito, M. Kuraguchi, Y. Takada, K. Tsuda, I. Omura, and T. Ogura, "Design optimization of high breakdown voltage AlGaN-GaN power HEMT on an insulating substrate for $\mathrm{R}_{\mathrm{ON}} \mathrm{A}-\mathrm{V}_{\mathrm{B}}$ tradeoff characteristics", Electron Devices, IEEE Transactions on, vol. 52, no. 1, pp. 106-111, 2005.

[6] X. Huili, Y. Dora, A. Chini, S. Heikman, S. Keller, and U. K. Mishra, "High breakdown voltage AlGaN-GaN HEMTs achieved by multiple field plates", Electron Device Letters, IEEE, vol. 25, no. 4, pp. 161-163, 2004.

[7] ATLAS User's Manual, Device Simulation Software, 2005ed Santa Clara, CA., SILVACO International, 2005. 